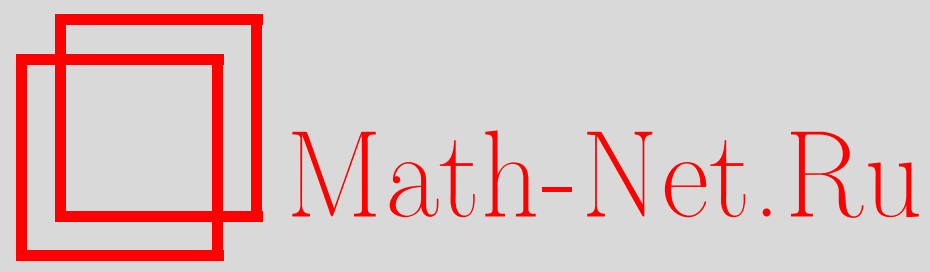

Д. Х. Муштари, Теорема Глисона для поля рациональных чисел и полей вычетов, Матем. заметки, 1998, том 64, выпуск 4, 584-591

DOI: https://doi.org/10.4213/mzm1433

Использование Общероссийского математического портала Math-Net.Ru подразумевает, что вы прочитали и согласны с пользовательским соглашением http://www . mathnet.ru/rus/agreement

Параметры загрузки:

IP : 107.22 .136 .117

26 апреля 2023 г., 14:54:11 


\section{ТЕОРЕМА ГЛИСОНА ДЛЯ ПОЛЯ РАЦИОНАЛЬНЫХ ЧИСЕЛ И ПОЛЕЙ ВЫЧЕТОВ}

\section{Д. Х. Муштари}

Рассматриваются заряды $\mu$ со значениями в поле $F$, заданные на ортомодулярных частично упорядоченных множествах (логиках), состоящих из всех проекторов, действующих в некотором конечномерном линейном пространстве над $F$. В случаях, когда $F$ - поле рациональных чисел или поле вычетов, доказьвается представление Глисона: $\mu(P)=\operatorname{tr}\left(T_{\mu} P\right)$, где $T_{\mu}-$ линейный оператор.

Библиография: 4 названия.

Рассматриваются заряды $\mu$ со значениями в поле $F$ на ортомодулярных частично упорядоченных множествах (логиках), состоящих из всех проекторов, действующих в некотором конечномерном линейном пространстве над $F$. Для некоторых полей $F$ доказьвается представление Глисона [1]:

$$
\mu(P)=\operatorname{tr}\left(T_{\mu} P\right)
$$

где $T_{\mu}-$ линейный оператор.

Классическая теорема Глисона устанавливает (1) для состояний (т.е. вероятностных мер), заданных на логике П $(H)$ всех эрмитовых проекторов на вещественном гильбертовом пространстве $H$ размерности не меньше 3. Представление (1) для зарядов в конечномерном случае требует условия ограниченности [2], иначе даже удовлетворяющий (1) заряд можно испортить суперпозицией с некоторой аддитивной, но неоднородной функцией на вещественной прямой. В бесконечномерном случае С. В. Дорофеев и А.Н. Шерстнёв [3] сняли условие ограниченности. В качестве области определения мер и зарядов можно рассматривать и другие логики, в частности, автор [4] рассмотрел логику $\mathfrak{P}(H)$ всех непрерывных (необязательно эрмитовых) проекторов на $H$. Доказанная в [4] теорема с учетом [3] может быть сформулирована следующим образом.

ТЕОрема 1. Пусть $H$ - бесконечномерное вещественное гильбертово пространство, $\mu: \mathfrak{P}(H) \rightarrow \mathbb{R}-$ функиия, сужение которой на любую $\sigma$-подалгебру счетно аддитивно. Тогда н удовлетворяет (1), где ядерный оператор $T_{\mu}$ единствен.

Из теоремы 1 следует, что на логике $\mathfrak{P}(H)$ нет ни одного состояния. Формулировка теоремы использует лишь язык логик и имеет смысл для многих других ситуаций, например, для зарядов на логике $\mathfrak{P}(X)$ всех непрерывных проекторов на некотором линейном топологическом (вещественном или $p$-адическом) пространстве $X$. Однако, доказательство таких обобщений приводит к пока еще непреодоленным трудностям. Дело

Работа выполнена при поддержке Российского фонда фундаментальных исследований, грант № 96-01-01265. 
в том, что доказательство теоремы 1 использует саму теорему Глисона применительно к подлогикам $\mathfrak{P}(H)$, изоморфньм $\Pi(H)$, каждый раз со своим оператором $T_{\mu}$, после чего доказьвается совпадение всех $T_{\mu}$. В бесконечномерном негильбертовом случае таких подлогик мало или нет, а в конечномерном случае теорема Глисона для зарядов неверна. Возникает вопрос: можно ли доказывать какие-либо аналоги теоремы 1 , не применяя при этом теорему Глисона? Любая аддитивная функция на поле $\mathbb{Q}$ рациональных чисел однородна, поэтому для конечномерных линейных пространств над $\mathbb{Q}$ отмеченньй вьше контрпример пропадает. Более того, верна следующая теорема.

TЕОрема 2. Пусть $\mathfrak{P}\left(\mathbb{Q}^{n}\right)$ - логика всех линейных проекторов на пространстве $\mathbb{Q}^{n}, n \geqslant 3, \mu: \mathfrak{P}\left(\mathbb{Q}^{n}\right) \rightarrow \mathbb{Q}-$ заряд, т.е. $\mu\left(P_{1}+P_{2}\right)=\mu\left(P_{1}\right)+\mu\left(P_{2}\right)$ для любих ортогональных $\left(P_{1} P_{2}=P_{2} P_{1}=0\right)$ проекторов $P_{1}$ и $P_{2}$ в $\mathfrak{P}\left(\mathbb{Q}^{n}\right)$. Тогда

$$
\mu(P)=\operatorname{tr}(T P) \quad \text { для всех } P \in \mathfrak{P}\left(\mathbb{Q}^{n}\right),
$$

әде линейный оператор Т определяется по $\mu$ единственным образом.

ДокАЗАтЕльство. Сначала рассмотрим случай $n=3$. Введем базис $e=(1,0,0)$, $f=(0,1,0), g=(0,0,1)$ в $\mathbb{Q}^{3}$ с биортогональным базисом $\left\{e^{\prime}, f^{\prime}, g^{\prime}\right\}$. Одномерные проекторы $P$ на $\mathbb{Q}^{3}$ представим в каноническом виде $N(P)^{-1} y^{\prime} \otimes y$ :

1) $N(P)^{-1} y^{\prime}(y)=1$;

2) каждый из векторов $y^{\prime}$ и $y$ имеет целые координаты, не имеющие общего множителя;

3) $N(P)$ - натуральное число, которое будем назьвать индексом проектора $P$.

По определению $y^{\prime} \otimes y: x \mapsto y^{\prime}(x) y$. Проекторы $N\left(P_{1}\right)^{-1} y_{1}^{\prime} \otimes y_{1}$ и $N\left(P_{2}\right)^{-1} y_{2}^{\prime} \otimes y_{2}$ ортогональны тогда и только тогда, когда $y_{1}^{\prime}\left(y_{2}\right)=y_{2}^{\prime}\left(y_{1}\right)=0$. С каждой тройкой попарно ортогональных одномерных проекторов $N\left(P_{1}\right)^{-1} y_{1}^{\prime} \otimes y_{1}, N\left(P_{2}\right)^{-1} y_{2}^{\prime} \otimes y_{2}, N\left(P_{3}\right)^{-1} y_{3}^{\prime} \otimes y_{3}$ (такие тройки будем называть реперными) ассоциируются две матрицы: $A\left(P_{1}, P_{2}, P_{3}\right)$, строки которой-векторы $y_{1}^{\prime}, y_{2}^{\prime}, y_{3}^{\prime}$, и $X\left(P_{1}, P_{2}, P_{3}\right)$, строки которой-векторы $y_{1}, y_{2}, y_{3}$.

Шаг 0. Введем проекторы $P_{1}, \ldots, P_{9}$, матрицы которьх определяют линейньй базис в девятимерном пространстве всех $3 \times 3$ рациональных матриц. Для определенности, базису $\{e, f, g\}$ в $\mathbb{Q}^{3}$ сопоставим базис из проекторов $\left\{P_{i}(e, f, g): 1 \leqslant i \leqslant 9\right\}$ :

$$
\begin{aligned}
& P_{1}=e^{\prime} \otimes e, \quad P_{2}=f^{\prime} \otimes f, \quad P_{3}=g^{\prime} \otimes g, \quad P_{4}=\left(e^{\prime}+f^{\prime}\right) \otimes e, \quad P_{5}=\left(e^{\prime}+g^{\prime}\right) \otimes e, \\
& P_{6}=\left(e^{\prime}+f^{\prime}\right) \otimes f, \quad P_{7}=\left(f^{\prime}+g^{\prime}\right) \otimes f, \quad P_{8}=\left(e^{\prime}+g^{\prime}\right) \otimes g, \quad P_{9}=\left(f^{\prime}+g^{\prime}\right) \otimes g .
\end{aligned}
$$

Предположим, $\mu$ удовлетворяет (2). Тогда оператор $T$ однозначно задается всеми значениями $\mu\left(P_{i}\right), 1 \leqslant i \leqslant 9$. Поэтому нам необходимо доказать лишь то, что любой заряд на $\mathfrak{P}\left(\mathbb{Q}^{3}\right)$ однозначно задается своими значениями на всех $P_{i}, 1 \leqslant i \leqslant 9$, а следовательно, совпадает с некоторьм зарядом, которьй имеет те же значения на $P_{i}$ и задается соотношением (2).

Воспользуемся равенствами

$$
\mu\left(P_{1}\right)+\mu\left(P_{2}\right)+\mu\left(P_{3}\right)-\mu\left(P_{1}^{\prime}\right)-\mu\left(P_{2}^{\prime}\right)-\mu\left(P_{3}^{\prime}\right)=0
$$

для всех реперных троек $\left\{P_{1}^{\prime}, P_{2}^{\prime}, P_{3}^{\prime}\right\}$. Фиксируем “область единственности" $\left\{P_{i}: 1 \leqslant\right.$ $i \leqslant 9\}$ и расширим ее до всей логики $\mathfrak{P}\left(\mathbb{Q}^{3}\right)$.

Назовем преобразование тройки векторов $\{x, y, z\}$ әлементарным, если оно получено последовательным применением нескольких преобразований вида:

1) замена одного вектора его разностью или суммой с другим вектором тройки;

2) перестановка векторов;

3) изменение знака одного из векторов. 
Каждому элементарному преобразованию базиса $\{e, f, g\}$ сопоставим әлементарное преобразование координат в пространствах $\mathbb{Q}^{3}$ и $\left(\mathbb{Q}^{3}\right)^{\prime}$. Сформулируем некоторые факты об элементарных преобразованиях координат $U$, которые нам потребуются.

ЛЕмма 1. Справедливы следующие утверждения:

а) $U$ - взаимно однозначное отображение иелочисленной решетки $\mathbb{Z}^{3} \subset \mathbb{Q}^{3}$ на себя;

б) свойство координат иелочисленного вектора в $\mathbb{Q}^{3}$ или в $\left(\mathbb{Q}^{3}\right)^{\prime}$ не иметь общий множстель сохраняется после применения $U$;

в) $U$ не меняет индекс проектора;

г) определитель, строки которого - координать векторов $x_{1}, x_{2}, x_{3}$ в $\mathbb{Q}^{3}$, совпадает по абсолютной величине с определителем, составленным из координат этих векторов после применения $U$, что верно и для векторов в сопряженном пространстве.

ЛЕМма 2. Пусть

$$
P_{1}=N\left(P_{1}\right)^{-1} y_{1}^{\prime} \otimes y_{1}, \quad P_{2}=N\left(P_{2}\right)^{-1} y_{2}^{\prime} \otimes y_{2}, \quad P_{3}=N\left(P_{3}\right)^{-1} y_{3}^{\prime} \otimes y_{3}
$$

- реперная тройка, причем $N\left(P_{1}\right)=p, N\left(P_{2}\right)=1, N\left(P_{3}\right)=p$. Тогда

$$
\left|\operatorname{det}\left(y_{1}, y_{2}, y_{3}\right)\right|=\left|\operatorname{det}\left(y_{1}^{\prime}, y_{2}^{\prime}, y_{3}^{\prime}\right)\right|=p \text {. }
$$

ДокАЗАТЕЛЬСТво. Из свойства 2) канонического вида проектора $P_{1}$ и утверждений б), в) леммы 1 легко следует, что существует такое элементарное преобразование координат, которое переводит $y_{1}^{\prime}$ в $(1,0,0)$, а $y_{1}$ в вектор с целыми координатами $(p, t, 0)$. При этом в силу ортогональности проекторов $P_{2}$ преобразуется к виду $(\alpha, \beta, \gamma) \otimes(0, \rho, \sigma)$, затем не меняющим вид $P_{1}$ элементарным преобразованием двух последних координат $P_{2}$ преобразуется к виду $(\alpha, \beta, 0) \otimes(0, \varphi, \psi)$. Согласно утверждению в) леммы 1 $\beta=\varphi=1$ и $P_{3}$ преобразуется в $p^{-1}(\tau, \pi, p) \otimes(0,0,1)$. Согласно утверждению г) леммы 1 оба определителя равны $p$.

Лемма 3. Если $N(P)=p q$, где р и в взаимно просты, то существует реперная троика $\left\{P, P_{1}, P_{2}\right\}$ такая, что $N\left(P_{1}\right)=p$ и $N\left(P_{2}\right)=q$.

ДокАЗАтЕльство. Элементарным преобразованием координат $P$ приводится к виду $(p q)^{-1}(1,0,0) \otimes(p q, s, 0)$, где $p q$ и $s$ взаимно просты. В новых координатах определим

$$
\begin{gathered}
P_{1}=N\left(P_{1}\right)^{-1}(-l s, l p q, n p) \otimes(0,1, p), \\
P_{2}=N\left(P_{2}\right)^{-1}(-s, p q,-q) \otimes(0, n,-l q), \quad n, l \in \mathbb{N} .
\end{gathered}
$$

Сушествуют целые $n$ и $l$ такие, что $n p+l q=1$. Тогда $N\left(P_{1}\right)=p, N\left(P_{2}\right)=q$.

Шаг 1. Обозначим через $\mathfrak{P}_{0}(e, f, g)$ множество всех проекторов, матрищы которых в базисе $\{e, f, g\}$ состоят только из чисел \pm 1 или 0 . Оно содержит все базисные проекторы $P_{i}$ и состоит из 87 элементов.

ЛЕмма 4. Если два заряда $\mu$ и $\nu$ совпадают на некоторых девяти линейно независимых әлементах в $\mathfrak{P}_{0}(e, f, g)$, то они совпадают на всем $\mathfrak{P}_{0}(e, f, g)$. 
ДОКАЗАТЕЛЬСТВО состоит в вычислении ранга системы всех уравнений вида (4), где тройки $\left\{P_{1}^{\prime}, P_{2}^{\prime}, P_{3}^{\prime}\right\}$ взяты из $\mathfrak{P}_{0}(e, f, g)$. С помощью компютера найдено, что число таких уравнений равно 105 и что ранг системы равен 78 (причем не равньй 0 определитель порядка 78 равен 1). Это доказьвает лемму.

Шаг 2 . Кроме $\left\{P_{i}(e, f, g): 1 \leqslant i \leqslant 9\right\}$ множество $\mathfrak{P}_{0}(e, f, g)$ содержит и другие базисы вида $\left\{P_{i}(x, y, z): 1 \leqslant i \leqslant 9\right\}$, определяемые тем же правилом, исходя из некоторых базисов $\{x, y, z\}$ в $\mathbb{Q}^{3}$. Используя это, докажем основную лемму.

ЛЕмма 5. Если заряд $\mu$ задан на девяти әлементах базиса $\left\{P_{i}(x, y, z): 1 \leqslant i \leqslant 9\right\}$, то этот заряд определен единственным образом на множестве $\mathfrak{P}(x, y, z ; \mathbb{Z})$ всех проекторов с целыми матричными әлементами по отношению к базису $\{x, y, z\}$.

ДокАЗАтЕльство. Без ограничения общности $x=e, y=f, z=g$. Покажем, что элементарными преобразованиями одного базиса в другой область единственности расширяется до множества $\mathfrak{P}(e, f, g ; \mathbb{Z})$.

Для определенности рассмотрим преобразование базиса $\{e, f, g\}$ в $\{e+f, f, g\}$ с биортогональным базисом $\left\{e^{\prime}, f^{\prime}-e^{\prime}, g^{\prime}\right\}$. Легко проверяется, что соответствующий (см. (3)) базис $\left\{P_{i}(e+f, f, g): 1 \leqslant i \leqslant 9\right\}$ также содержится в области единственности $\mathfrak{P}_{0}(e, f, g)$. В силу леммы 4 область единственности содержит также и 87 проекторов множества $\mathfrak{P}_{0}(e+f, f, g)$.

Итак, элементарные преобразования не вьводят из области единственности. Пусть $N(P)=1$, согласно лемме $3 P$ входит в реперную тройку $P=y^{\prime} \otimes y, P_{1}=y_{1}^{\prime} \otimes y_{1}$, $P_{2}=y_{2}^{\prime} \otimes y_{2}$. Достаточно показать, что элементарньм преобразованием базиса $\{e, f, g\}$ мы получим $\left(y, y_{1}, y_{2}\right)$. При элементарных преобразованиях базисов целочисленная матрица $X\left(P, P_{1}, P_{2}\right)$, составленная из векторов $\left(y, y_{1}, y_{2}\right)$, преобразуется с помощью элементарных преобразований строк.

Введем некоторое преобразование произвольной целочисленной матрицы $M$, являющееся результатом последовательности элементарных преобразований ее строк. Сначала обнуляются второй и третий элементы первого столбца, затем элементарными преобразованиями второй и третьей строк обнуляется третий элемент второго столбца. Далее диагональ приводится к неотрицательной, после чего все числа над диагональю делаются как можно меньше, но неотрицательными. Результат этой последовательности преобразований назовем редукиией матрицы $M$ и обозначим $\operatorname{red}(M)$.

Редукция свела матрицу со строками $y, y_{1}, y_{2}$ к целочисленной неотрицательной треугольной матрище. Согласно лемме 2 ее определитель равен 1. Поэтому на диагонали стоят 1 и ,на самом деле, получившаяся матрица единичная. Следовательно, соответствуюшим редукции преобразованием базис $\left\{y, y_{1}, y_{2}\right\}$ переводится в $\{e, f, g\}$. Последовательность обратных преобразований включает $P$ в область единственности.

Шаг 3. Обозначим через $\mathfrak{P}(p)$ множество всех проекторов $P$ таких, что $N(P)=p$. Всюду в этом разделе $p=q^{m}$, где $q$ - простое число. При продолжении области единственности на $\mathfrak{P}(p)$ возникают некоторые трудности. Например, рассмотрим $P=\left[p_{i j}\right]$ такой, что $p_{11}=1 / p$, где $p$ велико. Пусть $\mu$-глисоновский заряд с оператором $T_{\mu}=$ $P_{1}(e, f, g)$. Если мы докажем включение $\mathfrak{P}(p)$ в область единственности, то получим $\mu(P)=1 / p$. Однако, для доказательства этого равенства можно использовать лишь уравнения вида (4), т.е. линейные уравнения с коэффициентами \pm 1 или 0 при неизвестных, причем их правые части - целые числа (значения $\mu$ на тех проекторах, которые принадлежат $\mathfrak{P}(e, f, g ; \mathbb{Z}))$. Итак, из теоремы 2 , в частности, следует существование 
матрицы, состоящей из чисел \pm 1 или 0 , каждая строка которой задается некоторой реперной тройкой и определитель делится на $p$.

Лемма 6. Пусть $P=p^{-1}(a, b, c) \otimes(x, y, z), z$ не делится на q. Пусть проекторы $P, P_{1}=\left(a_{1}, b_{1}, c_{1}\right) \otimes\left(x_{1}, y_{1}, z_{1}\right), P_{2}=p^{-1}\left(a_{2}, b_{2}, c_{2}\right) \otimes\left(x_{2}, y_{2}, z_{2}\right)$ составляют реперную тройку. Тогда преобразование редукиии приводит матричу $A\left(P, P_{1}, P_{2}\right)$ $\kappa$ видy

$$
M(\alpha, \beta, p)=\left[a_{i j}\right]=\left(\begin{array}{ccc}
1 & 0 & \alpha \\
0 & 1 & \beta \\
0 & 0 & p
\end{array}\right),
$$

где $\alpha, \beta \in\{0,1,2, \ldots, p-1\}$ не зависят от выбора $P_{1}$ и $P_{2}$.

ДокАЗАТЕЛЬСТво. Покажем, что

$$
a_{11}=a_{22}=1 \text {. }
$$

Очевидно, что $(x, y, z)$ пропорционален вектору

$$
\left(\left|\begin{array}{ll}
b_{1} & c_{1} \\
b_{2} & c_{2}
\end{array}\right|,\left|\begin{array}{ll}
c_{1} & a_{1} \\
c_{2} & a_{2}
\end{array}\right|,\left|\begin{array}{ll}
a_{1} & b_{1} \\
a_{2} & b_{2}
\end{array}\right|\right) .
$$

Эти определители не могут одновременно делиться на $q$. Действительно, это означало бы, что две строки $\left(a_{1}, b_{1}, c_{1}\right)$ и $\left(a_{2}, b_{2}, c_{2}\right)$ пропорциональны $(\bmod q)$, причем по свойству 2) канонического представления коэффициент пропорциональности не может делиться на $q$. Однако, тогда равенство $a_{1} x_{1}+b_{1} y_{1}+c_{1} z_{1}=1$ и вытекающее из $P_{1} \perp P_{2}$ равенство $a_{2} x_{1}+b_{2} y_{1}+c_{2} z_{1}=0$ одновременно вьполняться не могут.

Итак, если $z$ не делится на $q$, то

$$
\left|\begin{array}{ll}
a_{1} & b_{1} \\
a_{2} & b_{2}
\end{array}\right| \neq 0(\bmod q) .
$$

Это означает, что матрица, составленная из двух первых столбцов матрицы $A\left(P, P_{1}, P_{2}\right)$, имеет ранг $(\bmod q)$ равньй 2 . Элементарные операции со строками не меняют этот ранг, в то же время, если неверно $(5)$, то ранг $(\bmod q)$ нашей матрицы равен 1 . Напомним (лемма 2$)$, что $\left|\operatorname{det}\left(A\left(P, P_{1}, P_{2}\right)\right)\right|=p$, поэтому $a_{11}=a_{22}=1, a_{33}=p$. Независимость чисел $\alpha, \beta$ от выбора $P_{1}$ и $P_{2}$ мы покажем ниже.

Далее, с каждым проектором $P \in \mathfrak{P}(p)$ будет ассоциирована реперная тройка $\left\{P, P_{1}\right.$, $\left.P_{2}\right\}$ из леммы 6. Обозначим через $\mathfrak{P}^{*}(i, j, k ; \alpha, \beta, p)$ (соответственно $\left.\mathfrak{P}_{*}(i, j, k ; \alpha, \beta, p)\right)$ множество всех $P$ таких, что после перестановки трех координат в порядке $i$-я, $j$-я, $k$-я и после редукции получим $\operatorname{red}\left(A\left(P, P_{1}, P_{2}\right)\right)=M(\alpha, \beta, p)\left(\operatorname{coответственно~} \operatorname{red}\left(X\left(P, P_{1}\right.\right.\right.$, $\left.\left.\left.P_{2}\right)\right)=M(\alpha, \beta, p)\right)$. Далее, для определенности рассмотрим случай $i=1, j=2, k=3$, а проектор $P$ возьмем из леммы 6. Легко видеть, что $\mathfrak{P}^{*}(1,2,3 ; \alpha, \beta, p)=\mathfrak{P}\left(e_{1}, f_{1}, g_{1} ; \mathbb{Z}\right)$, где $e_{1}=(1,0,0), f_{1}=(0,1,0), g_{1}=p^{-1}(-\alpha,-\beta, 1)$. Согласно лемме 5 для доказательства единственности заряда на всех элементах $\mathfrak{P}^{*}(1,2,3 ; \alpha, \beta, p)$ достаточно включить в область единственности следующие элементы базиса в $\mathfrak{P}^{*}(1,2,3 ; \alpha, \beta, p)$ :

$$
\begin{gathered}
P_{1}(\alpha, \beta)=(1,0, \alpha) \otimes(1,0,0), \quad P_{2}(\alpha, \beta)=(0,1, \beta) \otimes(0,1,0), \\
P_{3}(\alpha, \beta)=p^{-1}(0,0, p) \otimes(-\alpha,-\beta, 1), \quad P_{4}(\alpha, \beta)=(1,1, \alpha+\beta) \otimes(1,0,0), \\
P_{5}(\alpha, \beta)=(1,0, p+\alpha) \otimes(1,0,0), \quad P_{6}(\alpha, \beta)=(1,1, \alpha+\beta) \otimes(0,1,0), \\
P_{7}(\alpha, \beta)=(0,1, p+\beta) \otimes(0,1,0), \quad P_{8}(\alpha, \beta)=p^{-1}(1,0, p+\alpha) \otimes(-\alpha,-\beta, 1), \\
P_{9}(\alpha, \beta)=p^{-1}(0,1, p+\beta) \otimes(-\alpha,-\beta, 1) .
\end{gathered}
$$


Для вычисления $\alpha$ и $\beta$ выберем одну из координат ( $a$ или $b$ ) в каноническом представлении проектора $P$, которая не делится на $q$, и рассмотрим соответствующий столбец в матрице проектора $P$. Сопоставляя его с матричными элементами проекторов из базиса, мы видим, что если натуральное число $n$ удовлетворяет условию $1=n z(\bmod p)$, то $-\alpha=n x(\bmod p),-\beta=n y(\bmod p)$. Отсюда следует, что $\alpha$ и $\beta$ в лемме 6 не зависят от выбора $P_{1}$ и $P_{2}$.

Отметим, что лишь два элемента этого базиса, $P_{8}$ и $P_{9}$, принадлежат $\mathfrak{P}(p)$, остальные семь принадлежат $\mathfrak{P}(e, f, g ; \mathbb{Z})$, и согласно лемме 5 значение заряда на них определено единственньп образом. Рассмотрим матричные элементы $p_{i j}(k)$ проекторов $P_{k}$. Мы видим, что $p_{31}(k) \neq 0$ лишь для $k=8$ и $p_{32}(k) \neq 0$ лишь для $k=9$. Отсюда следует, что в разложении матрицы произвольного проектора $\left[p_{i j}\right] \in \mathfrak{P}^{*}(1,2,3 ; \alpha, \beta, p)$ по базисным элементам $P_{i}$ коэффициент при $P_{8}$ равен $p \cdot p_{31}$, а коэффициент при $P_{9}$ равен $p \cdot p_{32}$. То же верно и для $\mathfrak{P}_{*}(1,2,3 ; \alpha, \beta, p)$, в котором фиксирован базис из элементов $\left\{P_{i}^{\prime}(\alpha, \beta): 1 \leqslant i \leqslant 9\right\}$, отличающихся от элементов $P_{i}(\alpha, \beta)$ лишь порядком сомножителей в тензорном произведении.

Допустим, что $\alpha, \beta, \varphi, \xi$ - отличные от 0 целые числа такие, что

$$
1+\alpha \varphi+\beta \xi=p
$$

Рассмотрим проекторы

$$
\begin{gathered}
\pi_{1}=p^{-1}(\alpha, \beta, 1) \otimes(\varphi, \xi, 1), \quad \pi_{2}=p^{-1}(\alpha, \beta, 1) \otimes(\varphi+p \beta, \xi-p \alpha, 1), \\
\pi_{3}=p^{-1}(\alpha+p \xi, \beta-p \varphi, 1) \otimes(\varphi, \xi, 1), \quad \pi_{4}=p^{-1}(\alpha+p \beta, \beta, 1) \otimes(\varphi, \xi-p \varphi, 1) .
\end{gathered}
$$

Согласно приведенному вьше доказательству единственности в лемме 6 все $\pi_{i}$ принадлежат $\mathfrak{P}^{*}\left(1,2,3 ; p-\varphi^{\prime}, p-\xi^{\prime}, p\right) \cap \mathfrak{P}_{*}\left(1,2,3 ; p-\alpha^{\prime}, p-\beta^{\prime}, p\right)$, где $\alpha^{\prime}=\alpha(\bmod p)$, $\beta^{\prime}=\beta(\bmod p), \varphi^{\prime}=\varphi(\bmod p), \xi^{\prime}=\xi(\bmod p)$. Значения заряда $\mu$ на каждом $\pi_{i}$ можно выразить либо через его значения на базисных проекторах из $\mathfrak{P}^{*}\left(1,2,3 ; p-\varphi^{\prime}, p-\xi^{\prime}, p\right)$ (напомним, что из них лишь $P_{8}$ и $P_{9}$ не входят в $\mathfrak{P}(e, f, g ; \mathbb{Z})$ ), либо через его значения на базисных проекторах из $\mathfrak{P}_{*}\left(1,2,3 ; p-\alpha^{\prime}, p-\beta^{\prime}, p\right)$. Таким образом, мы получаем четыре уравнения относительно четырех неизвестных: $u=\mu\left(P_{8}\right)$ и $v=\mu\left(P_{9}\right)$, где $P_{8}, P_{9} \in \mathfrak{P}^{*}\left(1,2,3 ; p-\varphi^{\prime}, p-\xi^{\prime}, p\right)$, и $u^{\prime}=\mu\left(P_{8}^{\prime}\right)$ и $v^{\prime}=\mu\left(P_{9}^{\prime}\right)$, где $P_{8}^{\prime}, P_{9}^{\prime} \in \mathfrak{P}_{*}(1,2,3$; $\left.p-\alpha^{\prime}, p-\beta^{\prime}, p\right)$. Коэффициенты при неизвестных равны матричным элементам $p_{13}, p_{23}$, $p_{31}, p_{32}$ проекторов $\pi_{i}$. Вьпишем получившиеся уравнения:

$$
\begin{gathered}
\alpha u+\beta v-\varphi u^{\prime}-\xi v^{\prime}=c_{1}, \quad \alpha u+\beta v-(\varphi+p \beta) u^{\prime}-(\xi-p \alpha) v^{\prime}=c_{2}, \\
(\alpha+p \xi) u+(\beta-p \varphi) v-\varphi u^{\prime}-\xi v^{\prime}=c_{3}, \quad(\alpha+p \beta) u+\beta v-\varphi u^{\prime}-(\xi-p \varphi) v^{\prime}=c_{4},
\end{gathered}
$$

где числа $c_{i}$ вычисляются по значениям тех коэффициентов, которые соответствуют элементам базисов, принадлежащим $\mathfrak{P}(e, f, g ; \mathbb{Z})$. Определитель системы равен $-\varphi \beta p^{3} \times$ $(2 \alpha \varphi+2 \xi \beta)=-2 \varphi \beta p^{3}(p-1)$. Он отличен от 0 , так как $\varphi \neq 0, \beta \neq 0$. Поэтому однозначно определены значения заряда как на всех элементах $\mathfrak{P}^{*}\left(1,2,3 ; p-\varphi^{\prime}, p-\xi^{\prime}, p\right)$, так и на всех элементах $\mathfrak{P}_{*}\left(1,2,3 ; p-\alpha^{\prime}, p-\beta^{\prime}, p\right)$.

Легко показать, что если $\varphi^{\prime}$ не делится на $q$, а $\xi^{\prime}$ любое, то всегда можно найти $\varphi, \xi, \alpha, \beta$ такие, что имеет место $(6)$ и $\varphi^{\prime}=\varphi(\bmod p), \xi^{\prime}=\xi(\bmod p)$. Поэтому заряд однозначно задан на $\mathfrak{P}^{*}\left(1,2,3 ; p-\varphi^{\prime}, p-\xi^{\prime}, p\right)$. То же верно, если $\xi$ не делится на $q$. Если $\beta^{\prime}$ или $\alpha^{\prime}$ не делится на $q$, то заряд однозначно задан на $\mathfrak{P}_{*}\left(1,2,3 ; p-\alpha^{\prime}, p-\beta^{\prime}, p\right)$. 
Итак, если проектор $P$ из леммы 6 таков, что $c z$ не делится на $q$ (или $b y$, или $a x$ ), то по крайней мере одно из чисел $a x$ или $b y$ также не делится на $q$, согласно данному вьше описанию $P$ содержится в некотором $\mathfrak{P}^{*}\left(1,2,3 ; p-\varphi^{\prime}, p-\xi^{\prime}, p\right)$, причем хотя бы одно из чисел $\varphi^{\prime}, \xi^{\prime}$ не делится на $q$. Кроме того, $P$ принадлежит области единственности.

Несколько сложнее ситуация, когда в сумме $N(P)=a x+b y+c z$ каждое слагаемое делится на $q$. По определению троек $a, b, c$ и $x, y, z$ хотя бы одно из этих чисел не делится на $q$. Для определенности предположим, что это $z$. Тогда $P \in \mathfrak{P}^{*}(1,2,3 ; \alpha, \beta, p)$. Пусть $\alpha$ и $\beta$ делятся на $q$. Нужно показать, что заряд $\mu$ единствен на проекторах $P_{8}(\alpha, \beta)$ и $P_{9}(\alpha, \beta)$. Заменим эти проекторы на другие проекторы из $\mathfrak{P}^{*}(1,2,3 ; \alpha, \beta, p)$, которые вместе с остальными $P_{i}(\alpha, \beta)$ составляют базис, а именно, на

$$
\begin{gathered}
p^{-1}(1,-1, \alpha-\beta) \otimes(-\alpha,-\beta, 1) \in \mathfrak{P}_{*}(2,3,1 ; 1,-\alpha+\beta(\bmod p), p), \\
p^{-1}(1,1,2 p+\alpha+\beta) \otimes(-\alpha,-\beta, 1) \in \mathfrak{P}_{*}(2,3,1 ; p-1,-\alpha-\beta(\bmod p), p) .
\end{gathered}
$$

Согласно доказанному заряд единствен на этих проекторах, а следовательно, и на $P_{8}(\alpha, \beta)$ и $P_{9}(\alpha, \beta)$.

Итак, мы доказали единственность заряда для проекторов, индексы которых - степени простьх чисел. Благодаря лемме 3 к этому случаю сводится общий случай. Теорема 2 доказана для $n=3$.

Шаг 4. Теорема 2 для произвольного $n$ доказывается индукцией по $n$. Для простоты опишем лиш переход от $n=3$ к $n=4$.

Зададим проекторы

$$
\begin{aligned}
& e^{\prime} \otimes e=(1,0,0,0) \otimes(1,0,0,0), \quad f^{\prime} \otimes f=(0,1,0,0) \otimes(0,1,0,0), \\
& g^{\prime} \otimes g=(0,0,1,0) \otimes(0,0,1,0), \quad h^{\prime} \otimes h=(0,0,0,1) \otimes(0,0,0,1)
\end{aligned}
$$

и рассмотрим еще двенадцать базисных проекторов

$$
\begin{aligned}
& e^{\prime} \otimes(e+f), \quad e^{\prime} \otimes(e+g), \quad e^{\prime} \otimes(e+h), \quad f^{\prime} \otimes(f+e), \quad f^{\prime} \otimes(f+g), \quad f^{\prime} \otimes(f+h), \\
& g^{\prime} \otimes(g+e), \quad g^{\prime} \otimes(g+f), \quad g^{\prime} \otimes(g+h), \quad h^{\prime} \otimes(h+e), \quad h^{\prime} \otimes(h+f), \quad h^{\prime} \otimes(h+g) .
\end{aligned}
$$

Фиксируем заряд $\mu$ на этих проекторах. Из доказанного ранее следует, что заряд можно однозначно продолжить на все проекторы вида

$$
N(P)^{-1}\left(\varphi u^{\prime}+\eta v^{\prime}+\chi w^{\prime}\right) \otimes(\alpha u+\beta v+\gamma w)
$$

где $u, v, w$ - три произвольных вектора из канонического базиса $\{e, f, g, h\}$.

Возьмем проектор общего вида $\left(\varphi e^{\prime}+\eta f^{\prime}+\chi g^{\prime}+\tau h^{\prime}\right) \otimes(\alpha e+\beta f+\gamma g+\delta h)$. Он содержится в линейной оболочке девяти линейно независимых проекторов

$$
\begin{gathered}
N(P)^{-1}\left(\varphi e^{\prime}+\eta f^{\prime}\right) \otimes(\alpha e+\beta f), \quad g^{\prime} \otimes g, \quad h^{\prime} \otimes h, \\
N(P)^{-1}\left(\varphi e^{\prime}+\eta f^{\prime}\right) \otimes(\alpha e+\beta f+g), \quad N(P)^{-1}\left(\varphi e^{\prime}+\eta f^{\prime}\right) \otimes(\alpha e+\beta f+h), \\
g^{\prime} \otimes(\alpha e+\beta f+g), \quad g^{\prime} \otimes(g+h), \quad h^{\prime} \otimes(\alpha e+\beta f+h), \quad h^{\prime} \otimes(g+h),
\end{gathered}
$$

причем каждьй из этих проекторов удовлетворяет условию (7). Таким образом, заряд любого четырехмерного проектора однозначно определен.

Точно так же осуществляется переход от размерности $n$ к $n+1$. 
ЗАмЕчАНИЕ. В действительности, на первом, втором и четвертом шагах мы доказали теорему 2 для логики $\mathfrak{P}\left(\mathbb{Z}^{n}\right)$.

Сформулируем теорему 2 в алгебраических терминах.

ТЕОРема 3. Если $n \geqslant 3$, то любая рациональнозначная аддитивная функиия на множестве всех идемпотентов в $\operatorname{Hom}\left(\mathbb{Q}^{n}, \mathbb{Q}^{n}\right)$ имеет аддитивное продолэсение на все $\operatorname{Hom}\left(\mathbb{Q}^{n}, \mathbb{Q}^{n}\right)$.

ЗАдАчА 1 . Пусть $R, R_{1}$ - два кольца, $\mathfrak{P}$ - логика всех идемпотентов в кольце $R$. В каком случае любой аддитивньй оператор $\mu: \mathfrak{P} \rightarrow R_{1}$ допускает аддитивное продолжение на все $R$ ?

КромеколецHom $\left(\mathbb{Q}^{n}, \mathbb{Q}^{n}\right)$ и $\operatorname{Hom}\left(\mathbb{Z}^{n}, \mathbb{Z}^{n}\right)$ это утверждение доказано для кольца всех операторов в конечномерном пространстве над полем вычетов (для них повторяется доказательство леммы 5 , причем существенно используется, что детерминант ненулевого определителя на первом шаге можно выбрать равным 1). Таким образом, верна

ТЕОрема 4. Пусть $F$ - поле вычетов по простому модулю $p, n \geqslant 3, \mathfrak{P}\left(F^{n}\right)-$ логика всех линейных проекторов на пространстве $F^{n}, \mu: \mathfrak{P}\left(F^{n}\right) \rightarrow F-$ заряд. Тогда

$$
\mu(P)=\operatorname{tr}(T P) \quad \text { для всех } P \in \mathfrak{P}\left(F^{n}\right),
$$

где линейный оператор Т определяется по н единственным образом.

Решение задачи 1 для операторов в линейных пространствах над произвольным полем характеристики 0 позволило бы получить, используя счетную аддитивность, аналоги теоремы Глисона для некоторых бесконечномерньх линейных топологических пространств над полем вешественных или $p$-адических чисел.

ЗАДАчА 2. Интересно доказать аналог теоремы 2 для логики $\Pi\left(\mathbb{Q}^{3}\right)$ всех проекторов, которые являются эрмитовыми по отношению к каноническому скалярному произведению, заданному базисом $\{e, f, g\}$.

К сожалению, использованный в настоящей статье прием перехода от одного базиса к другому в этой ситуации не работает.

\section{СПИСОК ЦИТИРОВАННОЙ ЛИТЕРАТУРЫ}

[1] Gleason A. M. Measures on the closed subspaces of a Hilbert space // J. Math. Mech. 1957. V. 6. №6. P. 885-893.

[2] Шерстнёв А.Н. Понятие заряда в некоммутативной схеме теории меры // Вероятностные методы и кибернетика. №10-11. Казань, 1974. С. 68-72.

[3] Дорофеев С. В., Шерстнёв А.Н. Функции реперного вида и их применения // Изв. вузов. Матем. 1990. № 4. С. 23-29.

[4] Муштари Д.Х. Логики проекторов в банаховых пространствах // Изв. вузов. Матем. 1989. № 8 . C. 44-52. 